\title{
Leak Detection Modeling and Simulation for Oil Pipeline with Artificial Intelligence Method
}

\author{
Pudjo Sukarno ${ }^{1}$, Kuntjoro Adji Sidarto ${ }^{2}$, Amoranto Trisnobudi ${ }^{3}$, Delint Ira \\ Setyoadi $^{2}$, Nancy Rohani ${ }^{2} \&$ Darmadi $^{4}$ \\ ${ }^{1}$ Faculty of Earth Sciences and Mineral Technology \\ ${ }^{2}$ Faculty of Mathematics and Natural Sciences \\ ${ }^{3}$ Faculty of Industrial Technology \\ ${ }^{4}$ Research Consortium OPPINET - ITB
}

\begin{abstract}
Leak detection is always interesting research topic, where leak location and leak rate are two pipeline leaking parameters that should be determined accurately to overcome pipe leaking problems. In this research those two parameters are investigated by developing transmission pipeline model and the leak detection model which is developed using Artificial Neural Network. The mathematical approach needs actual leak data to train the leak detection model, however such data could not be obtained from oil fields. Therefore, for training purposes hypothetical data are developed using the transmission pipeline model, by applying various physical configuration of pipeline and applying oil properties correlations to estimate the value of oil density and viscosity. The various leak locations and leak rates are also represented in this model. The prediction of those two leak parameters will be completed until the total error is less than certain value of tolerance, or until iterations level is reached. To recognize the pattern, forward procedure is conducted. The application of this approach produces conclusion that for certain pipeline network configuration, the higher number of iterations will produce accurate result. The number of iterations depend on the leakage rate, the smaller leakage rate, the higher number of iterations are required. The accuracy of this approach is clearly determined by the quality of training data. Therefore, in the preparation of training data the results of pressure drop calculations should be validated by the real measurement of pressure drop along the pipeline. For the accuracy purposes, there are possibility to change the pressure drop and fluid properties correlations, to get the better results. The results of this research are expected to give real contribution for giving an early detection of oil-spill in oil fields.
\end{abstract}

Keywords: pipeline leak detection; transmission pipeline; artificial intelligence.

\section{$1 \quad$ Introduction}

\subsection{Background}

Leaking in pipeline network in Indonesian oil fields are becoming and increasingly serious issues especially in old oil fields, either onshore or 
offshore. Considering the environmental issues, leak problems should be avoided by conducting leak detection, either in terms of amount of leakage or the leak locations. There should be some methods to determine those two leak parameters, as soon as possible, that could be used as operational parameters to overcome pipe leaking problems. This research would cover wide ranges of pipe leaking problems, especially for leak location and leakage rate.

\subsection{Objective}

The main objectives of this research project are developing method to estimate leak location and leakage rate, by developing model to simulate leak detection in oil transmission pipeline using a new approach based on Artificial Neural Network. The model then further develops as software, which could be used to determine the leak(s) location and the leakage rate. The results of this research are expected to give real contribution for giving an early detection of oil-spill in oil fields:

\subsection{Scope of Work}

The scope of work of these research activities consists of:

- Literature study, which emphasized in the leak models developments. The supporting methods that are related to the development of leak models had also been studied, such as fluid flow in pipes which represent pipeline flow for single phase that is oil flow. This study also includes gathering information from oil fields, which have pipeline system. The information from oil fields enriched the model and represents the main conditions of pipeline.

- Mathematical modeling development; there are two models which had been prepared in this research that are the oil pipeline model and the leak detection model.

The oil pipeline model is basically pipeline network model, which simulates the oil flow in pipeline network and predict the pressure distribution along the pipe without and with leaking conditions, at a certain flow condition. The leak is represented by a branch of pipe, with certain diameter. The pipe branch could be located at any point along the main pipe, and under certain leakage rate, the pressure distributions along the pipe are calculated. Using this model, the pressure distribution, with or without leaks, is determined. The model produces enough data for any leak conditions, and the data will be used to train the leak detection simulator.

The leak detection model is formulated based on the mathematical formulation, based on the artificial neural network. According to the artificial neural network method, the model should be trained using the actual leaking data. Since, such kind of data is not available in oil field in a large numbers, than hypothetical leaks data are developed, using the oil 
pipeline model. The detail development will be discussed in the next chapter.

- Generating hypothetical data and collecting data from field; the hypothetical data had been generated using the oil pipeline model, with various conditions of pipeline and leaking problems. The actual field data for leak problems are not easy to find, but there is one leak problem that has been recorded in an offshore oilfield. This data will be used for validation purposes.

- Software development to predict the leak problems, based on the results of oil pipeline model, and the mathematical modeling, the software to predict the leak problems was developed. The software consists of leak detection model, and sub-routine which contains pipeline leaks hypothetical data which are generated using the oil pipeline model.

- The model validation had been conducted by comparing the field data and hypothetical data to the calculation result using the software. The prepared hypothetical data for validation are developed using the oil pipeline model, which are different with the hypothetical data for training purposes.

\subsection{Report Organization}

This paper consists of four sections. Section 1 is this introductory note. Section 2 reviews the existing methodologies. Preliminary study for leaking pipeline presented in Section 3, and Section 4 presents the proposed method from this research.

\section{Review of Previous Leak Detection Methods}

Many papers have been published as results of researches concerning leak detection in pipeline. Those papers had been studied, as part of this research activity, and a brief review about the previous publication of leak detection methods are presented in this paper. Based on the papers that had been studied, the leak detection methods can be classified into three categories, that is:

- hardware based methods

- biological methods

- $\quad$ software based methods

Schematically the pipeline leak detection methods could be discussed as shown in Figure 1, where the methodology of each method is presented.

Every leak detection method, which will be presented on this Section, was developed using different approaches, therefore every of method has advantages and disadvantages. For comparing the performance of those methods, it is 
necessary to define the key attributes of a leak detection problem. The parameters that were used to assess leak detection methods are as follows:

- Leak sensitivity

- Location estimating capability

- Operational change

- Availability

- False alarm rate

- Maintenance requirement

- Cost

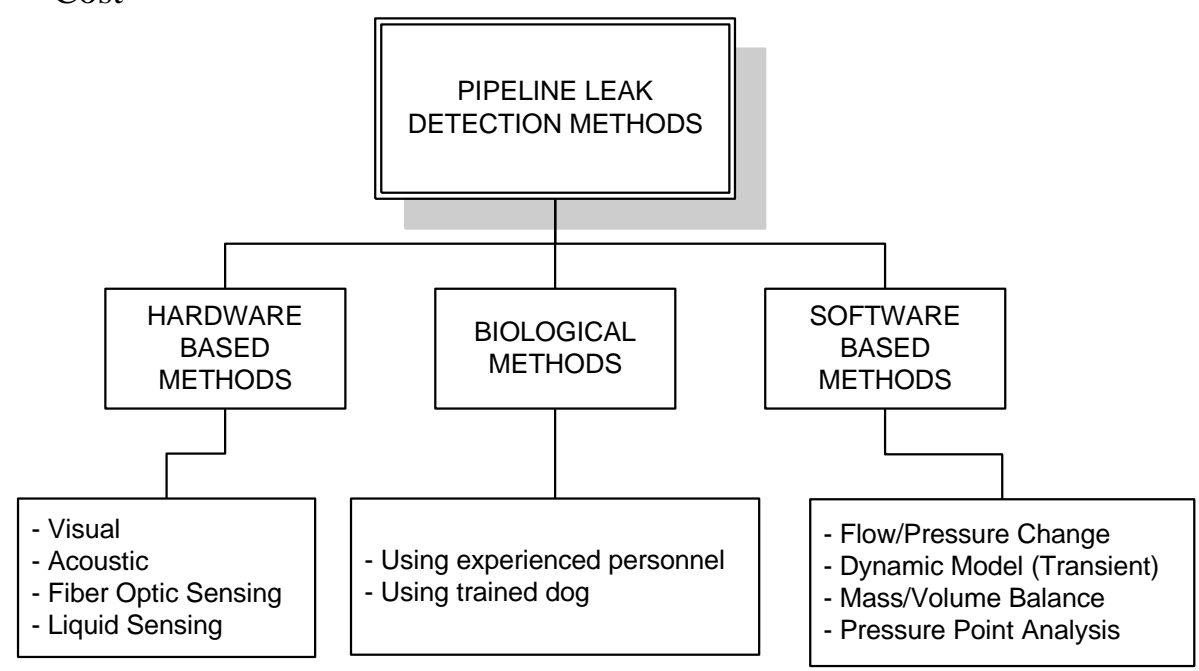

Figure 1 Leak Detection Methods Categories.

Based on those parameters, the comparison between every method is shown in the following Table 1 .

Based on the comparisons it could be concluded, that most of the methods were developed based on the phenomena of fluid flow in pipes, and human resources dependent. In this research, it is quite close to Biological Approach, by using the phenomena of fluid flow in pipes, but the leak detection is determined by using the software that developed based on the artificial neural network. For detail discussions about these methodologies, please refer to [1].

\section{The Development of Mathematical Modeling}

The first stage in these research activities is the model development of leak problem in an oil pipeline. The model will be developed by taking into consideration that a leak is treated as an outlet segment, in a part of a pipeline network. The outlet segment has certain diameter and very short length. In the 
model, the diameter of the outlet segment will represent the size of leak, where the diameter could be adjusted to represent the leak opening. The length of outlet segment is taken short, short enough to make the pressure drop effect along the short segment is negligible, compared with the pressure drop along the main line. A mathematical model for the network pipeline will be represented by single phase (oil) pressure drop equation in a pipeline, Kirchoff's law for representing the network pipeline, Swamee and Jain's Correlation for friction factor determination. The oil properties which are included in the pressure drop calculation will be estimated by using Standing's Correlation for calculating oil density and Glasso's Correlation for determining oil viscosity. Based on this model, the leak in the oil pipeline could be simulated, with various leaking conditions, which are the leak location and the leaking rate of oil. In a real life, it is quite difficult to locate the leak point, when it happened. However, based on the result of pipeline simulation, the pressure distribution in the pipeline due to leak problems show certain patterns, which could be used as references for determining the leak location in the pipeline. The pressure distribution patterns could be used to predict the location of pipeline leaks by using Artificial Neural Network Method, where this method replicates human intelligence. The method could be trained to identify the leak location based on the pattern of pressure distribution in the pipeline, due to leak problems.

Table 1 The Comparison of Leak Detection Method.

\begin{tabular}{c|cccccccc}
\hline Method & Biological & Visual & Acoustic & $\begin{array}{c}\text { Flow/Pres } \\
\text { change }\end{array}$ & $\begin{array}{c}\text { Mass/vol. } \\
\text { balance }\end{array}$ & $\begin{array}{c}\text { Dynamic } \\
\text { model }\end{array}$ & PPA & $\begin{array}{c}\text { Liquid } \\
\text { sensing }\end{array}$ \\
\hline $\begin{array}{c}\text { Leak } \\
\text { Sensitivity }\end{array}$ & yes & yes & yes & no & no & yes & yes & yes \\
$\begin{array}{c}\text { Location } \\
\text { estimate }\end{array}$ & yes & yes & yes & no & no & yes & no & yes \\
$\begin{array}{c}\text { Operational } \\
\text { change }\end{array}$ & yes & yes & no & no & no & yes & no & no \\
$\begin{array}{c}\text { Availability } \\
\text { FALSE } \\
\text { alarm }\end{array}$ & no & no & yes & yes & yes & yes & yes & yes \\
$\begin{array}{c}\text { Maintenance } \\
\text { requirement }\end{array}$ & medium & medium & medium & low & low & high & medium & low \\
Cost & high & high & medium & low & low & high & medium & high \\
\hline
\end{tabular}

The following discussion concerning the model development will be conducted in detail, which will be used as the main references in the model development. 


\subsection{Artificial Neural Network Method}

Artificial Neural Network Method (ANN) is a relatively new method that tries to replicate human intelligence. By giving ANN some sets of data, it can recognize patterns in those data. This method represents a significant departure from the conventional approach to model development. In this model a set of equations are not necessary required, but the method will process the provided data concerning the pressure distribution in the pipeline which has leaking problems. The pressure distributions are represented by the input and the output conditions in the pipeline that consist of pressure and flow rate.

The conventional mathematical and statistical model links the inputs to the outputs via explicit equations. Therefore if the equations are not correct, or if the parameters are incorrect, or if the functions are miss-specified, then the conventional model will not work. The task is hard because the model development has to be done by experts and often their models become compromises due to lack of knowledge or to simplify the problems due to lack of data or reliable computational tractability. The neural network performs the same function as the conventional model but the ANN model should learn to relate the inputs to the outputs without being given any explicit equations.

The ANN is trained to recognize patterns of pressure distribution, that will be used as references to locate the leaking point. This involves providing the network with input values for which the corresponding output value is known. The desired result is then 'back-propagated' from the output nodes through the intermediate nodes to the input nodes. The weight values are modified to encourage the network to produce the right output for the input supplied. In this way, the weight values come to represent patterns in the data.

Among the different Artificial Intelligence structures, Back Propagation Neural Network (BPNN) by Rumelhart, as mention by Freeman, et.al.[3] is the most popular one because of its applicability in many different areas. Figure 2 shows common BPPN architecture.

Suppose there are $\mathrm{p}$ pairs of vectors, $\mathbf{x}_{1}, \mathbf{y}_{1}, \mathbf{x}_{2}, \mathbf{y}_{2}, \ldots, \mathbf{x}_{p}, \mathbf{y}_{p}$, with $\mathbf{x}_{i} \in \mathbf{R}^{n}$ and $\mathbf{y}_{i} \in \mathbf{R}^{m}$, and mapping $\Phi$ of $\mathbf{x}$ to $\mathbf{y}$ such that $\Phi \mathbf{x}_{i}=\mathbf{y}_{i}$ is implemented, then value of a unit is obtained as a non-decreasing and differentiable function (called an activation function) of a linear combination of the unit inputs. In practice, the sigmoid activation function is usually used for BPNN:

$$
f n e t=\frac{1}{1+e^{-n e t}}
$$




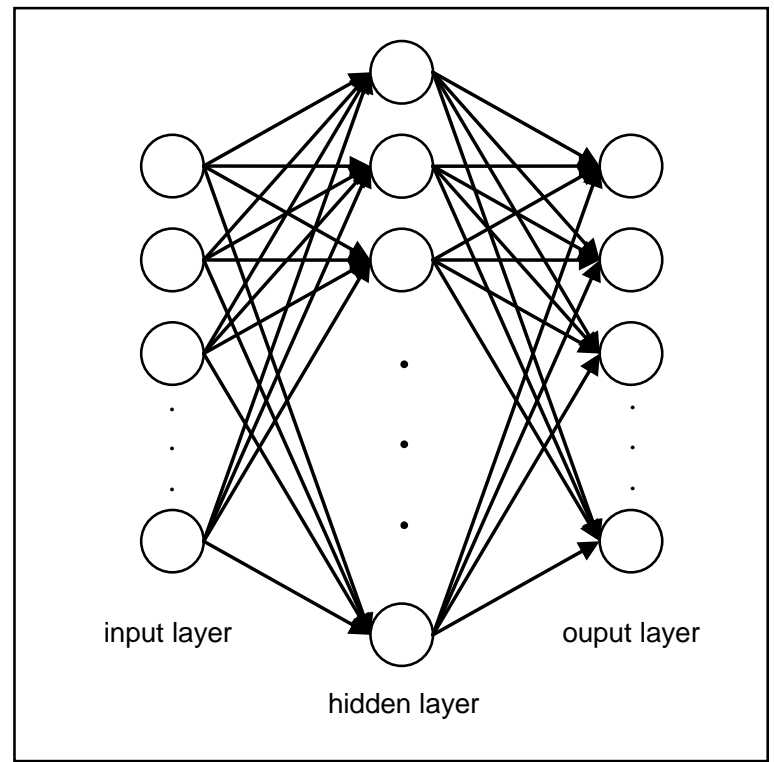

Figure 2 Common BPNN Architecture.

The following are training procedure for BPNN using gradient descent:

1. Initialize all weights and unit to small random values.

2. For all training pattern $\mathrm{p}$ :

\section{Feed Forward}

- Apply the input vector $x_{1, p}, x_{2, p}, \ldots, x_{N_{1}, p}$ to the input units (first layer).

- For layer $n=2,3, \ldots, L$ :

○ For unit $i=1,2, \ldots, N_{n}$ :

- Calculate the net-input values:

$$
n e t_{i, n}=\sum_{j=1}^{N_{p-1}} w_{j i, n} o_{j, n-1}
$$

- Calculate the outputs:

$$
o_{i, n}=f \text { net }_{i, n}
$$

- Calculate the error terms:

$$
E_{p}=\frac{1}{2} \sum_{i=1}^{N_{L}} y_{i, p}-o_{i, L}
$$




\section{Backpropagation}

- Calculate the unit error:

○ For layer $n=L, L-1, \ldots, 2$ :

- For unit $i=1,2, \ldots, N_{n}$ :

if $n=L$

$$
\delta_{i, n}=o_{i, n} 1-o_{i, n} \quad y_{i, p}-o_{i, n}
$$

else

$$
\delta_{i, n}=o_{i, n} 1-o_{i, n} \sum_{j=1}^{N_{p+1}} w_{i j, n+1} \delta_{j, n+1}
$$

- Update weights:

○ For layer $n=2,3, \ldots, L$ :

- For unit $i=1,2, \ldots, N_{n-1}$ :

- For unit $j=1,2, \ldots, N_{n}$ :

- Compute the weight increments:

$$
\Delta w_{i j, n} t=\eta \delta_{j, n} o_{i, n-1}+\alpha \Delta w_{i j, n} t-1
$$

- Compute the new values of the weights:

$$
w_{i j, n} t=w_{i j, n} t+\Delta w_{i j, n} t-1
$$

3. Compute the total error:

$$
E=\sum_{p} E_{p}
$$

The training will be completed until the total error is less than certain value of tolerance, or until some iteration level is reached. To recognize the pattern, forward procedure is conducted.

Because the characteristic of ANN is to recognize pattern of pressure distribution in the pipeline which has leaking problem, then the ANN could also be used to detect leak. By training the machine about the condition of normal (without leaking conditions) and abnormal (with leaking conditions) pressure distribution data, then the leak location could be detected, when a leak problem occurred. If information about location and leak rate which are related to the abnormal pressure distribution data is being given on the training phase, the method would not only able to predict the occurrence of leak, but also able to predict the location of leaking point, if a set of actual pressure distribution data is given. 
ANN requires as many pressure distribution data under leakage condition as possible for its training phase. The prepared data for training would be better if it is originated from the real field data. Unfortunately, such data are difficult to obtain. Therefore, for these training purposes the pressure distribution data with leaking conditions were generated using the pipeline model that is the pressure point analysis method.

\subsection{The Pressure Point Analysis Method}

The basic flow equation of oil in pipeline is used in the development mathematical model of fluid flow in pipe. The model will be used to predict the pressure distribution along the pipe under normal condition (without leaking condition).

The further derivation of the mathematical model yields a system of equations which are consists of three continuity equations. For solving the problems the following assumptions are implied:

1. the study considers that the main pipeline is treated as one segment of pipe,

2. there is only one leak in the pipeline,

3. the flow is steady state, horizontal,

4. the flow could be laminar or turbulent, and

5. the flow condition is isothermal.

Derivation of the developed model can be explained by considering one segment of pipe, as shown in Figure 3.

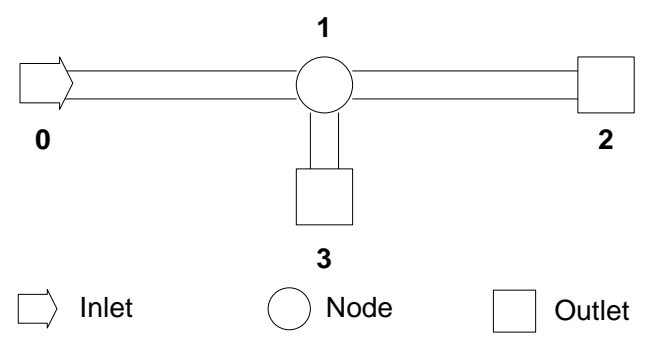

Figure 3 A Pipeline Segment with One Leak.

The pressure drop of oil in a segment of horizontal pipe, which has L in length and $\mathrm{d}$ in diameter, can be expressed as follow:

$$
\Delta P=97.10^{-4} \frac{\rho f \gamma q^{2} L}{d^{5}}
$$


Considering a simple pipeline network as shown in Figure 3 and in normal condition (without leak in pipe), the pipeline consists of the $0-2$ segment, and the segment $1-3$, is neglected. At this condition, the oil pressure drop equation along the segment of the pipe is represented by

$$
\Delta P_{02}=97.10^{-4} \frac{\rho f \gamma q_{0}^{2} L}{d^{5}}
$$

If a leak is exists in the pipeline, where the location and the size of leak is represented as a branch in the main pipe to form a network pipeline, then the pipeline network configuration could be divided into three segments, that is segment $0-1,1-2$ and $1-3$, as shown in Figure 3 . The liquid pressure drop equation for each segment is represented by the following equation:

$$
\begin{aligned}
& \Delta P_{01}=97.10^{-4} \frac{\rho f \gamma q_{0}{ }^{2} x}{d^{5}}, \\
& \Delta P_{12}=97.10^{-4} \frac{\rho f \gamma\left(q_{0}-q_{\text {leak }}\right)^{2}(L-x)}{d^{5}},
\end{aligned}
$$

and

$$
\Delta P_{13}=97.10^{-4} \frac{\rho f \gamma q_{\text {leak }}^{2} L_{\text {leak }}}{d_{\text {leak }}^{5}}
$$

In there is a leak in the pipeline, the leak is considered as a new segment, where in Figure 3, it is shown as segment 1-3. The segment that representing leak, is assumed to have outlet pressure, $P_{3}$, equal to atmospheric pressure i.e. 14.7 psia. For the case of offshore pipeline, the outlet pressure could be estimated by the sea hydrostatic pressure, which is depending on the depth of the seafloor of the sea bottom. In this paper the values of $L_{\text {leak }}, d_{\text {leak }}$ and $f_{\text {leak }}$ are assumed to be very small values. The assumptions are $1 \mathrm{ft}$ for $L_{\text {leak }}$ and 1 inch for $d_{\text {leak }}$. Because $f_{\text {leak }}$ is a function from $\varepsilon_{\text {leak }} / d_{\text {leak }}$, the value of $\varepsilon_{\text {leak }} / d_{\text {leak }}$ would be smaller than $\varepsilon / d$ of the pipeline, in order to neglect the friction factor. For the segment 1-3 in Figure 3, the friction factor is

$$
f_{\text {leak }}^{-0.5}=1.14-2 \log \left[\frac{\varepsilon_{\text {leak }}}{d_{\text {leak }}}+\frac{21.25}{N_{\mathrm{Re}}^{0.9}}\right]
$$

where $\varepsilon_{\text {leak }} / d_{\text {leak }}$ is chose as a value, which is smaller than $\varepsilon / d$. 
For oil flow rate distribution on each node, use the mass balance between incoming rates to the outgoing rates. For example, consider node 1, based on Kirchoff's law the summation of all flow rate has to be satisfied, that follow equation (7):

$$
q_{0}=q_{\text {leak }}+q_{2}
$$

In the equations (2), (3) and (4), the values of all variables are known, except for $P_{1}$ and $x$. By solving these equations, the unknown variables can be determined.

For solving the equations, the oil properties should be calculated. Since, the oil properties are depend on pressure and temperature, then the available correlation, such as for calculating the oil density Standing's correlation is used. Similarly the Glaso's correlation is applied to determine the oil viscosity. Each of the correlation is as follows:

\section{Standing's Correlation}

The oil density, at a given pressure and temperature, is determined using the Standing's Correlation (1981), which can be written as:

$$
\rho=\frac{62.4 \gamma}{0.972+0.0001471 .25 T^{1.175}}
$$

where $\gamma$ is oil specific gravity at standard conditions, as a function of ${ }^{\circ} \mathrm{API}$, denoted by:

$$
\gamma=\frac{141.5}{A P I+131.5}
$$

\section{Glasso's Correlation}

The oil viscosity is determined using Glasso's Correlation as follows:

$$
\mu=3.141 .10^{10} T^{-3.444}[\log A P I]^{a}
$$

where

$$
a=10.313[\log T]-36.447
$$


The basic oil properties data which are required to calculate pressure drop consists of ${ }^{\circ} \mathrm{API}$ gravity of oil, besides pressure and temperature at the intake of pipe.

For oil flow in pipes, where the pipe has $\mathrm{D}$ in diameter and $\mathrm{L}$ in length and if the oil has certain density and velocity, then, in general, the pressure drop along the pipe could be represented by the following equation:

$$
\Delta p=\frac{1}{2} \frac{L}{D} f \rho v^{2}
$$

If $i$ and $j$ representing intake point and discharge point, respectively, at a certain segment of pipe, then equation (12) could be written as the following equation:

$$
p_{i}-p_{j}=\frac{1}{2} \frac{L_{i j}}{D_{i j}} f_{i j} \rho v_{i j}^{2}
$$

It is understood that the direction of flow is from the high to low pressure point, and the other major constraint is that mass must be conserved. Therefore, the net flow into each node must be zero. In equation (12), $v$ is the average velocity, and should be related to the mass or volume flow rate. The mass flow rate can be expressed as

$$
m=\rho Q=\rho v A
$$

where: $A=\pi \frac{D^{2}}{4}$, the cross sectional area of the pipe, and $\mathrm{Q}$ is the volume flow rate. Using equation (14), $v$ in equation (13) can be represented in term of Q, and by substituting the area of pipe cross section, the previous equation in pressure drop equation could be redefined as follows:

$$
p_{i}-p_{j}=8 \frac{L_{i j} f_{i j} \rho Q_{i j}^{2}}{\pi^{2} D^{5}}
$$

The friction factor, $\mathrm{f}_{\mathrm{ij}}$, in equation (15) could be determined based on Swamee and Jain's Correlation, which is depending on the value of Reynold's Number. The equation for calculating the Reynold's Number is as follows:

$$
N_{\mathrm{Re}}=\frac{92.1 \cdot \gamma \cdot q}{\mu \cdot d}
$$

For turbulent flow i.e. $5000<N_{\mathrm{Re}}<10^{8}$, the friction factor can be predicted by using the following correlation: 


$$
f^{-0.5}=1.14-2 \log \left[\frac{\varepsilon}{d}+\frac{21.25}{N_{\mathrm{Re}}^{0.9}}\right]
$$

Based on equation (15), the flow rate could be determined, and produces the following equation:

$$
Q_{i j}= \pm \sqrt{\left|p_{i}-p_{j}\right|\left(\frac{\pi^{2} D_{i j}^{5}}{8 f_{i j} \rho L_{i j}}\right)}
$$

Simplifying the equation for flow rate, yields the following equation:

$$
Q_{i j}=\frac{\left(p_{i}-p_{j}\right) \sqrt{\frac{\pi^{2} D_{i j}^{5}}{8 f_{i j} \rho L_{i j}}}}{\sqrt{\left|p_{i}-p_{j}\right|}}
$$

If, $c_{\mathrm{ij}}$, is defined as:

$$
c_{i j}=\sqrt{\frac{\pi^{2} D_{i j}^{5}}{8 f_{i j} \rho L_{i j}\left|p_{i}-p_{j}\right|}}, \text { this equation could be simplified and produces }
$$

the following equation:

$$
c_{i j}=\sqrt{\frac{D_{i j}^{5}}{11.5 \times 10^{-5} f_{i j} L_{i j}\left|p_{i}-p_{j}\right|}}
$$

Substituting equation (20) to the equation (18) then mass flow rate equation can be written in simplified form as shown in equation (21).

$$
Q_{i j}=c_{i j} p_{i}-p_{j}
$$

Therefore, using equation (21), the conservation of mass at node $\mathrm{j}$ can be written as:

$$
\sum_{i} Q_{i j}=0
$$

where the summation is over all nodes $i$ that connect to node $j$. In general case,

$$
\sum_{i} c_{i j} p_{i}-p_{j}=0
$$


Thus, at each node where the pressure is unknown, then the pressure could be represented by,

$$
p_{j}=\frac{\sum_{i} c_{i j} p_{i}}{\sum_{i} c_{i j}}
$$

Once the pressures are determined the flow rates can be found from equation (19).

\section{Development of the Proposed Leak Detection Method}

After completing the mathematical model development, the main software that consists of two parts, which are the artificial neural network and the pressure point analysis, are developed. The development of the main software, including generating the data for training, has been successfully conducted, and in this Chapter the performances of the main software are shown.

Based on the pre-determined pipeline model as shown in Figure 3, this model will be tested to estimate the location of leak position. At the first stage, using the pressure point analysis method, data generation for training the model is conducted. The following procedure is conducted in generating the data.

1. Divide pipeline into $(n-1)$ equal segment with $\mathrm{n}$ nodes. If node $i$ and $j$ are connected by a segment, define a $I_{i j}=1$ and $I_{i j}=0$, otherwise.

2. Assume initial values of pressure.

3. Estimate $f_{i j}$, the estimation is not critical, but don't use zero.

4. Re-arrange equation (23) to solve for the unknown pressures in equation (24)

5. Compute the flow rates from equation (19)

6. Compute friction factor from equation (17)

7. Compute the updated values of $c_{i j}$

8. Go to step 3 and repeat steps 3-6 iteratively until the changes in inlet volume flow rate are smaller than some predetermined tolerance.

Based on the procedure to generate the data for training, a set of pipeline configuration is introduced. Table 2 shows range of pipeline data that consists of flow rate, inlet pressure, fluid properties, pipe length and diameter.

The length of pipe is divided into 10 nodes, and leak locations are varied and positioned on node. Therefore, for 10 nodes, there are 10 possibilities of leak location that each leak position will produce certain pressure distribution. The example results of data generation are shown in Table 3, that shows the intake 
and discharge pressure, for several pipe diameter (12", 16", 20", and 24"), and leak position, which covers wide ranging of pipe length. The leakage rates are also varied, as shown in the first column in Table 3.

Table 2 Basic Data to Generate Training Data.

\begin{tabular}{llr}
\hline Number of nodes & $:$ & 10 \\
Input flow rate & $:$ & $28000 \mathrm{bpd}$ \\
Inlet pressure & $:$ & $300 \mathrm{psia}$ \\
Viscosity & $:$ & $1.5 \mathrm{Cp}$ \\
Roughness & $:$ & $0.0005 \mathrm{~m}$ \\
Segment length & $:$ & $80,60,40 \mathrm{~km}$ \\
Pipe diameter & $:$ & $12 \%, 16 ”, 20 ", 24 ”$ \\
\hline
\end{tabular}

Using the prepared data the software is trained to determine the leak location. After training, the Artificial Neural Network would be able to predict the position of leak based on the information which has been fed into the software. There is some consideration during training stages that is the total error (the difference between ANN predictions with the data sample). If the total error decreases, it could be concluded that the prediction approaches the right answer. On the other way, if the total error is large but decreases, the calculation could be continued by applying higher number of iterations.

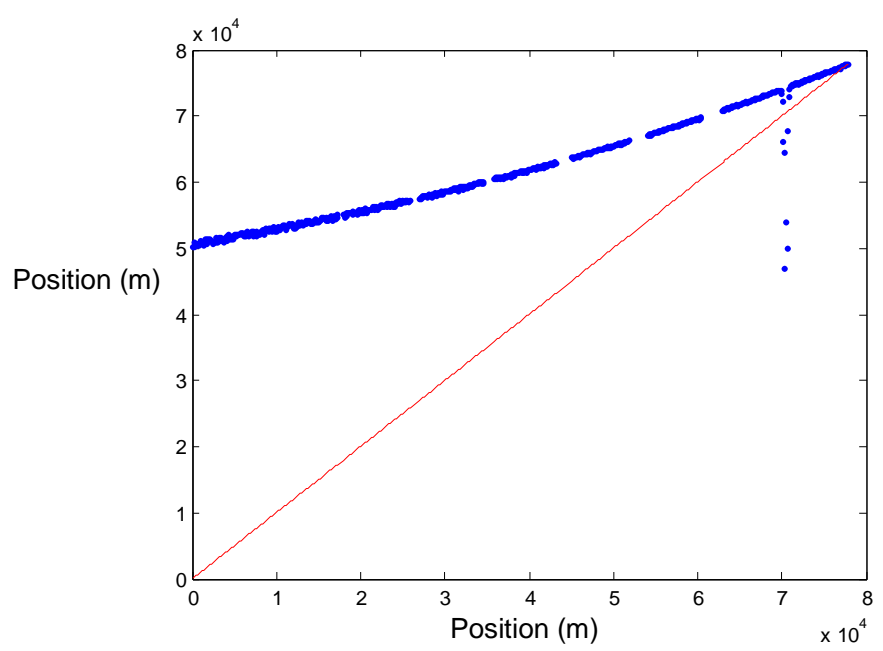

Figure 4 The Comparison between ANN Prediction and Actual Data For $80 \mathrm{~km}$ length of pipe and 24" of outside diameter by $1 \%$ leak rate (Number of Iteration $10,000)$. 
Table 3 Example of Data for Training.

\begin{tabular}{|c|c|c|c|c|c|c|c|c|c|}
\hline \multirow[t]{2}{*}{ Leakage } & \multicolumn{2}{|c|}{24} & \multicolumn{2}{|c|}{20} & \multicolumn{2}{|c|}{16} & \multicolumn{2}{|c|}{12} & \multirow{2}{*}{$\begin{array}{c}\text { Position } \\
100\end{array}$} \\
\hline & 300.03337 & 294.28556 & 300.80047 & 287.05242 & 300.85008 & 260.75205 & 300.89646 & 140.50742 & \\
\hline & 300.48608 & 294.73814 & 300.02242 & 286.27407 & 300.98488 & 260.88595 & 300.81615 & 140.42345 & 200 \\
\hline & 300.13578 & 294.38771 & 300.37693 & 286.62826 & 300.94857 & 260.84873 & 300.54843 & 140.15206 & 300 \\
\hline & 300.77467 & 295.02647 & 300.94478 & 287.19581 & 300.61643 & 260.51568 & 300.85525 & 140.45521 & 400 \\
\hline & 300.75654 & 295.00822 & 300.8577 & 287.10842 & 300.56091 & 260.45925 & 300.18666 & 139.78296 & 500 \\
\hline & 300.93902 & 295.17473 & 300.47637 & 286.68875 & 300.59114 & 260.37697 & 300.25315 & 139.39529 & 12800 \\
\hline & 300.86472 & 295.1003 & 300.35045 & 286.56252 & 300.57656 & 260.36147 & 300.90524 & 140.04371 & 12900 \\
\hline & 300.97776 & 295.21321 & 300.76662 & 286.97837 & 300.37331 & 260.15732 & 300.90477 & 140.03957 & 13000 \\
\hline & 300.13018 & 294.3655 & 300.85986 & 287.07131 & 300.78461 & 260.56772 & 300.09029 & 139.22143 & 13100 \\
\hline & 300.38588 & 294.62108 & 300.76241 & 286.97355 & 300.96902 & 260.75121 & 300.22256 & 139.35003 & 13200 \\
\hline & 300.29461 & 294.50369 & 300.85164 & 287.00002 & 300.26199 & 259.86003 & 300.7438 & 139.12791 & 33300 \\
\hline & 300.37509 & 294.58404 & 300.36373 & 286.51179 & 300.75034 & 260.34747 & 300.64129 & 139.02174 & 33400 \\
\hline & 300.77251 & 294.98133 & 300.41665 & 286.56441 & 300.34618 & 259.9424 & 300.01034 & 138.38712 & 33500 \\
\hline & 300.36932 & 294.57801 & 300.79393 & 286.94138 & 300.3502 & 259.94551 & 300.56653 & 138.93965 & 33600 \\
\hline \multirow[t]{15}{*}{$1 \%$} & 300.84685 & 295.05541 & 300.29449 & 286.44162 & 300.11677 & 259.71117 & 300.01675 & 138.3862 & 33700 \\
\hline & 300.68921 & 294.89764 & 300.00254 & 286.14937 & 300.48912 & 260.08261 & 300.3735 & 138.73928 & 33800 \\
\hline & 300.35068 & 294.55899 & 300.17616 & 286.32268 & 300.00281 & 259.59539 & 300.39405 & 138.75617 & 33900 \\
\hline & 300.76186 & 294.94445 & 300.10511 & 286.18983 & 300.22886 & 259.64012 & 300.28885 & 137.91901 & 53600 \\
\hline & 300.91904 & 295.1015 & 300.56001 & 286.64442 & 300.62851 & 260.03885 & 300.9312 & 138.5577 & 53700 \\
\hline & 300.69851 & 294.88084 & 300.01676 & 286.10086 & 300.18086 & 259.5903 & 300.13067 & 137.75349 & 53800 \\
\hline & 300.81998 & 295.00217 & 300.2172 & 286.30099 & 300.62601 & 260.03454 & 300.28522 & 137.90438 & 53900 \\
\hline & 300.28383 & 294.4659 & 300.44541 & 286.52889 & 300.54715 & 259.95477 & 300.11712 & 137.73262 & 54000 \\
\hline & 300.20974 & 294.39167 & 300.80158 & 286.88475 & 300.62159 & 260.0283 & 300.49083 & 138.10266 & 54100 \\
\hline & 300.08565 & 294.23644 & 300.85635 & 286.86469 & 300.81028 & 259.99742 & 300.09971 & 136.82521 & 78100 \\
\hline & 300.22498 & 294.37565 & 300.46015 & 286.46819 & 300.64483 & 259.83105 & 300.26385 & 136.98569 & 78200 \\
\hline & 300.57011 & 294.72065 & 300.79493 & 286.80265 & 300.97235 & 260.15768 & 300.51953 & 137.2377 & 78300 \\
\hline & 300.74084 & 294.89125 & 300.42417 & 286.43158 & 300.35794 & 259.54235 & 300.88837 & 137.60288 & 78400 \\
\hline & 300.37761 & 294.52789 & 300.74681 & 286.75391 & 300.72764 & 259.91114 & 300.09892 & 136.80976 & 78500 \\
\hline & 300.01475 & 294.1649 & 300.35018 & 286.35697 & 300.28705 & 259.46964 & 300.07134 & 136.77851 & 78600 \\
\hline
\end{tabular}

The prediction of leak location is shown on Figure 4, where the blue dots are obtained from the ANN software, and the red dots are the target values from the data.

Figure 4 shows that there are big difference between ANN prediction and the actual data, based on number of iteration 10,000. Increasing number of iteration would improve the accuracy. In this research the number of iteration is increased up to 20,000 iterations and the result is shown in Figure 5. Based on 
those two results, it could be concluded, that number of iterations has strong influence on the accuracy of leak location prediction. If the number of iterations is increased, the computing time will also increase. The approximate computing time for 20,000 iterations is approximately 1 hour.

Similar to Figures 4 and 5 for various conditions of pipeline and leak point locations, with different values of pipe diameter, and leakage rate had also been conducted, but it does not included in this paper.

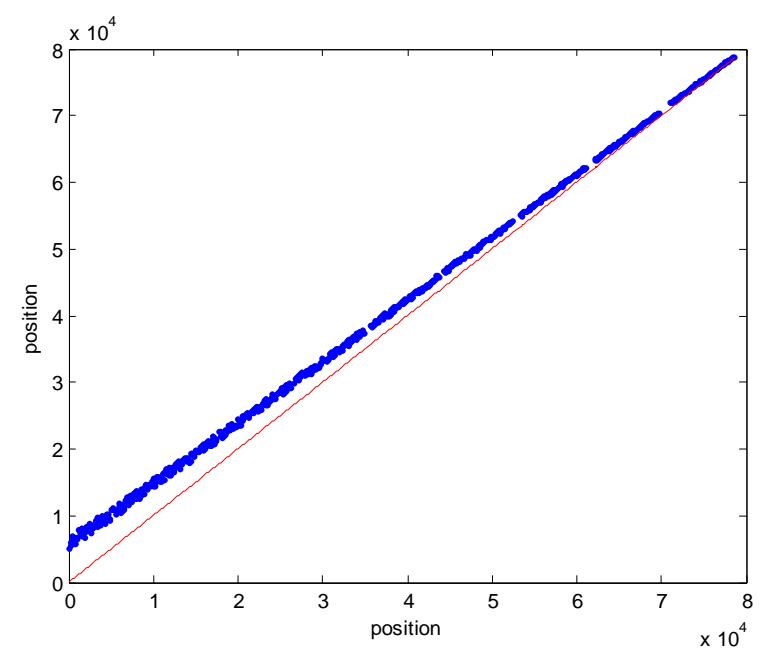

Figure 5 The Comparison between ANN Prediction and Actual Data For $80 \mathrm{~km}$ length of pipe and 24 " of outside diameter by $1 \%$ leak rate (Number of Iteration 20,000)

\section{Conclusion}

- The accuracy to predict the leak location depends on the variety of data which are provided for the training. In this case the variety of data consists of pipe length and diameter, flow rate, intake pressure, and discharge pressure. If the input is not in the range of data for training, then the results would be inaccurate.

- In case the real field data concerning the pressure distribution due to leak problems were not available, then the data for training may be generated using the network pipeline model, based on the existing pipeline network and the actual oil properties. The leak locations are defined hypothetically and the pressure point method is used to calculate the pressure distribution. Since the generated data were developed based on certain configuration of pipeline network, then the Artificial Neural Network method could only solve the problems for that certain configuration. So, if the pipeline has 
different configuration, then the data training could not be used anymore. In other words, if the pipe configuration is changed, then the new data training should be prepared.

- The accuracy of leak location prediction is also determined by the number of iteration. Higher number of iterations would produce more accurate solutions.

- For wider coverage of solving problems, variety of data could be applied when developing data for training. The input data to generate pressure distribution are not only limited to the existing configuration of pipeline network, but the input data could be extended to cover different diameters of pipeline, intake and discharge pressures, lengths of pipe, and flow rates. The artificial neural network method would be able to determine the leak location for pipeline configuration in the range of the input data. However, the training time would be longer, since the training process covers a lot number of data.

\section{$6 \quad$ Suggestion}

- The data preparation for training the software, depend on the complexity of network pipeline configuration. For simple network pipeline, as shown in this example, takes some times to prepare the data. For higher complexity of pipeline configuration it would take longer time. Therefore, there should be better approach in generating data for training for higher complexity of network pipeline configuration.

- The proposed method has limited ability that it works only for certain pipeline configuration. In the real conditions, there are many possibilities of pipeline configuration. Based on this real condition, there should be certain data for training that would be prepared. Further researches should be conducted to find dimensionless groups of variable that could represent any kind of pipeline network configuration, which become the general data that will be provided for the training. If it is possible, then general software to determine the leak location in a pipeline network could be developed, using a general data for training.

\section{Acknowledgement}

The authors are grateful to the Osaka Gas Foundation by providing the research funds in the year 2006, so this research had been conducted successfully. Our gratitude for the OPPINET of ITB to allow us to use the computer facilities. 


\section{References}

[1] Sukarno, P., et al., Development of an Accurate Pipeline Leak Detection Method, Oppinet 3rd Annual Report, 2004.

[2] Sukarno, P., et al., Development of Artificial Neural Network and Pressure Point Analysis Methods to Detect Leakage in Multi-phase Flow in Simple Pipeline, Oppinet 4th Annual Report, 2005.

[3] Freeman, James A., Skapura, David M., Neural Networks: Algorithms, Applications, and Programming Techniques, Addison Wesley, United States of America, 1992.

[4] Caputo, Antonio C., Pelagagge, Pacifico M., An Inverse Approach For Piping Networks Monitoring, 4th International Conference on Inverse Problems in Engineering, 2002. 